Supporting Information

\title{
Synthesis, Photochromic Properties, and Crystal Structures of Salts Containing a Pyridinium-Fused Spiropyran: Positive and Negative Photochromism in the Solution and Solid State
}

Yusuke Funasako,* Haruka Miyazaki, Takuro Sasaki, Kenta Goshima, and Makoto Inokuchi

Department of Applied Chemistry, Faculty of Engineering, Sanyo-Onoda City University, 1-1-1, Daigakudori,

Sanyo-Onoda, Yamaguchi 756-0884, Japan

E-mail: funasako@rs.socu.ac.jp

Table of Contents

Figure S1. Synthetic scheme for [6'-MC]X.

Figure S2. Kinetics plot of thermal coloring process of [ $\left.\mathbf{6}^{\prime}-\mathrm{SP}\right] \mathrm{I}$.

Figure S3. Experimental and TD-DFT calculated absorption spectra of [6'-MC]I and [5'-MC]I.

Figure S4. Photographs of crystals of $\left[6^{\prime}-\mathrm{SP}^{\prime}\right] \mathrm{PF}_{6}$ and $\left[6^{\prime}-\mathrm{MC}\right] \mathrm{PF}_{6}$ obtained by recrystallization.

Figure S5. Raman and reflectance IR spectra of the $\left[6^{\prime}-{ }^{\prime}-P^{\prime}\right] \mathrm{PF}_{6}$ single crystal before and after UV irradiation.

Figure S6. PXRD patterns of $\left[6^{\prime}-\mathrm{MC}\right] \mathrm{X}$.

Table S1. Crystallographic parameters for $\left[6^{\prime}-\mathrm{MC}\right] \mathrm{I},\left[\mathbf{6}^{\prime}-\mathrm{MC}\right] \mathrm{PF}_{6}$, and [6' $\left.-\mathrm{SP}\right] \mathrm{PF}_{6}$.

Tables S2-S3. Cartesian coordinates and energies of the optimized geometries of $\left[6^{\prime}-\mathbf{M C}\right]^{+},\left[6^{\prime}-\mathbf{S P}\right]^{+},\left[\mathbf{5}^{\prime}-\mathbf{M C}\right]^{+}$, and $\left[\mathbf{5}^{\prime}-\mathbf{S P}\right]^{+}$.

Table S4. Electronic excitation energies, oscillator strengths, and compositions of $\left[\mathbf{6}^{\prime}-\mathbf{M C}\right]^{+}$and $\left[\mathbf{5}^{\prime}-\mathbf{M C}\right]^{+}$ calculated by TD-DFT calculations.

References 

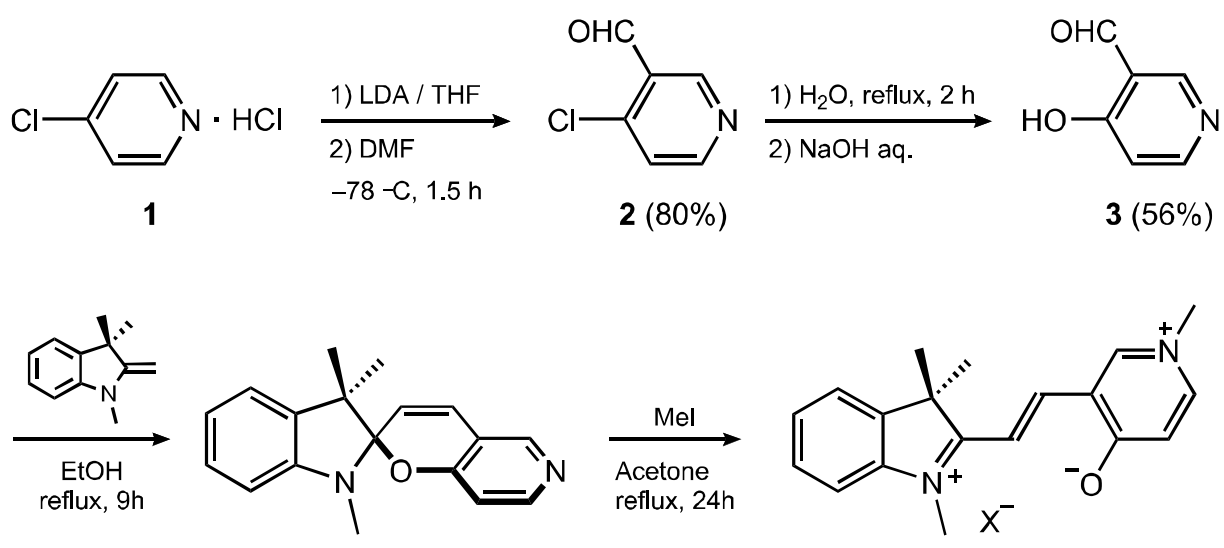

$4(83 \%)$

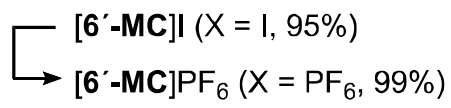

Figure S1. Synthetic scheme for [6'-MC]X.

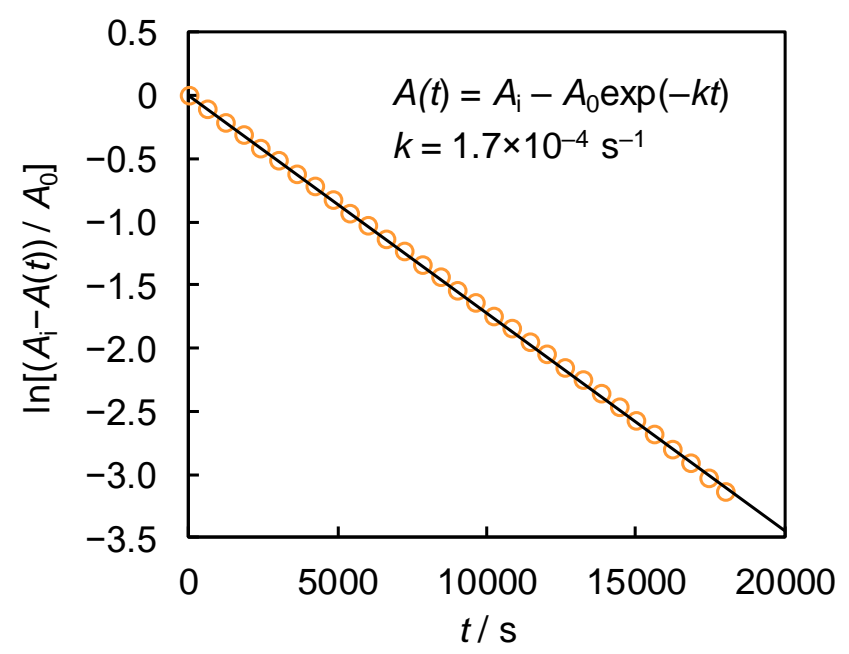

Figure S2. Kinetics plot of the thermal coloring process of $\left[6^{\prime}-\mathrm{SP}\right] \mathrm{I}$ (monitored at $458 \mathrm{~nm}$ ) in $\mathrm{CHCl}_{3}$ after - min irradiation with visible light at $25^{\circ} \mathrm{C}$. 
(a)

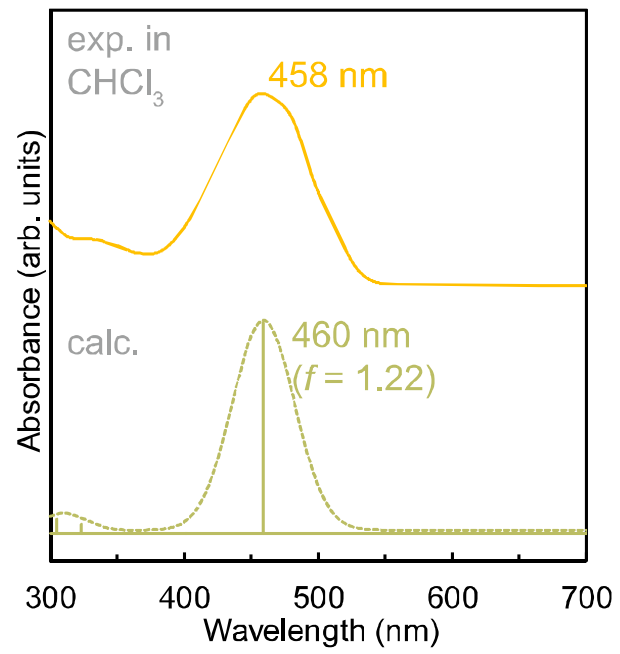

(b)

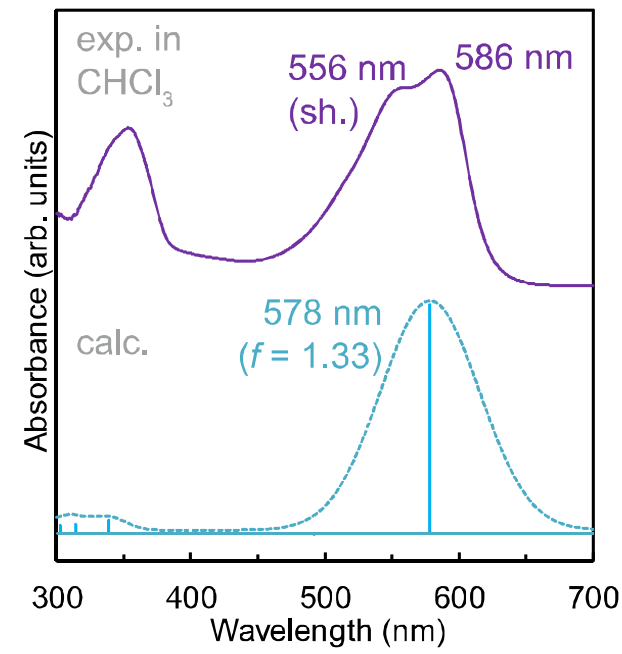

Figure S3. Experimental (top) and TD-DFT (CAM-B3LYP/def2-SVP) calculated (bottom) absorption spectra of (a) [6'-MC]I and (b) [5'-MC]I. The calculated spectra were shifted by $0.6 \mathrm{eV}$ toward lower energies ${ }^{\mathrm{S} 1}$.

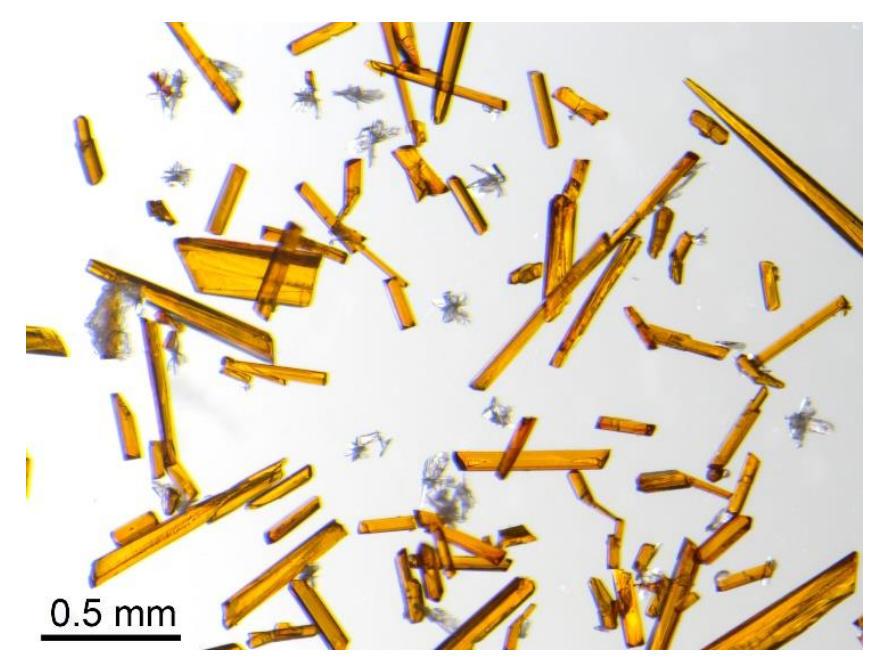

Figure S4. Photographs of the prismatic colorless crystals of [6' $-\mathbf{S P}] \mathrm{PF}_{6}$ were deposited together with orange needles of $\left[\mathbf{6}^{\prime}-\mathbf{M C}\right] \mathrm{PF}_{6}$ by recrystallization from acetone/ $\mathrm{Et}_{2} \mathrm{O}$. 

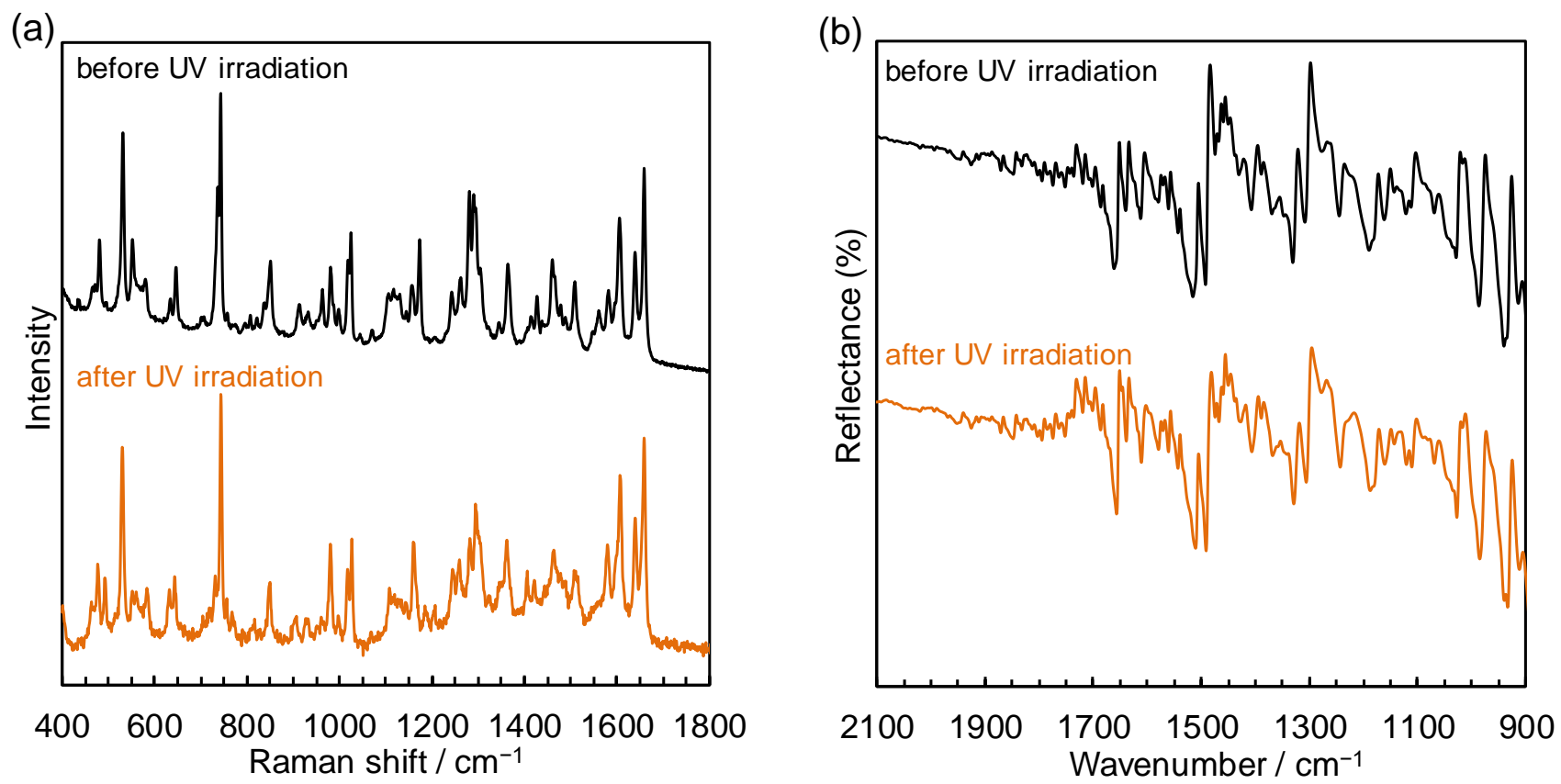

Figure S5. (a) Raman and (b) reflectance IR spectra of the $\left[6^{\prime}-\mathbf{S P}\right] \mathrm{PF}_{6}$ single crystal under the microscope before (black line) and after (orange line) UV light irradiation for $5 \mathrm{~min}$.

(a)

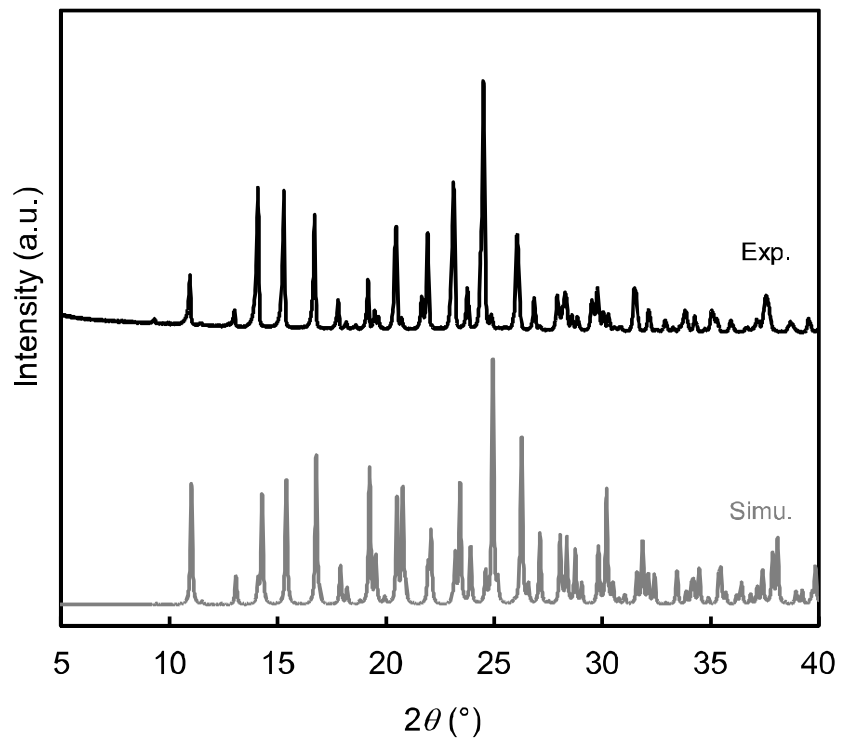

(b)

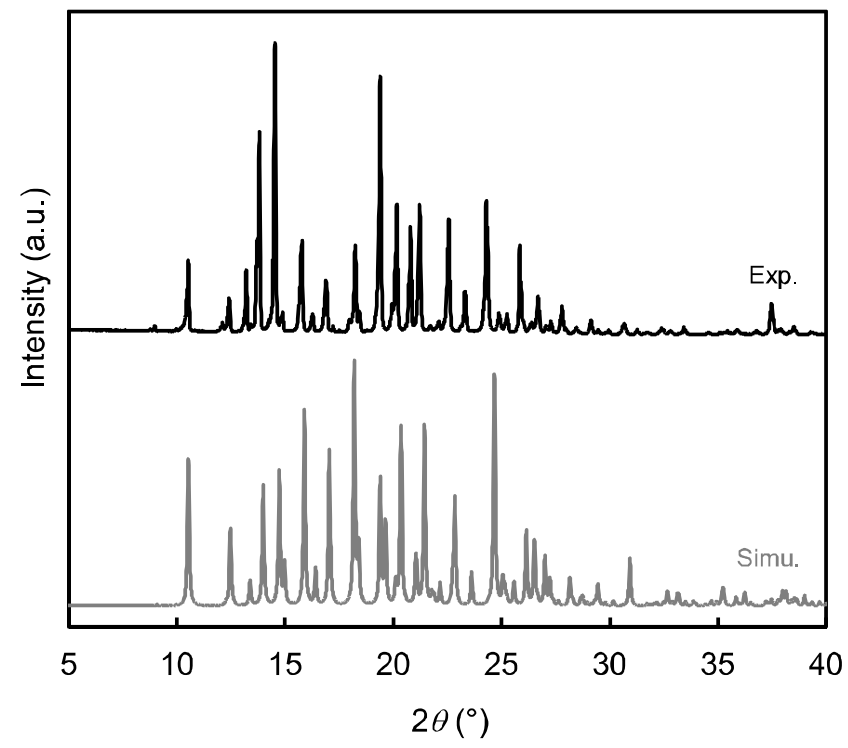

Figure S6. PXRD profiles of (a) $\left[\mathbf{6}^{\prime}-\mathrm{MC}\right] \mathrm{I}$ and (b) $\left[\mathbf{6}^{\prime}-\mathbf{M C}\right] \mathrm{PF}_{6}$. The experimental (black lines, upper) and the simulation pattern from the CIF (gray lines, bottom) are displayed. 
Table S1. Crystallographic parameters for $\left[6^{\prime}-\mathbf{M C}\right] \mathrm{I},\left[6^{\prime}-\mathbf{M C}\right] \mathrm{PF}_{6}$, and $\left[6^{\prime}-\mathrm{SP}\right] \mathrm{PF}_{6}$.

\begin{tabular}{|c|c|c|c|}
\hline & {$\left[6^{\prime}-\mathrm{MC}\right] \mathrm{I}$} & {$\left[6^{\prime}-\mathrm{MC}^{\prime} \mathrm{PF}_{6}\right.$} & {$\left[6^{\prime}-\mathrm{SP}\right] \mathrm{PF}_{6}$} \\
\hline empirical formula & $\mathrm{C}_{19} \mathrm{H}_{21} \mathrm{IN}_{2} \mathrm{O}$ & $\mathrm{C}_{19} \mathrm{H}_{21} \mathrm{~F}_{6} \mathrm{~N}_{2} \mathrm{OP}$ & $\mathrm{C}_{19} \mathrm{H}_{21} \mathrm{~F}_{6} \mathrm{~N}_{2} \mathrm{OP}$ \\
\hline formula weight & 420.28 & 438.35 & 438.35 \\
\hline$T / \mathrm{K}$ & 100 & 100 & 100 \\
\hline crystal system & orthorhombic & orthorhombic & monoclinic \\
\hline space group & $P 2_{1}{ }_{1} 2_{1}$ & $P 2_{1} 2_{1} 2_{1}$ & $P 2_{1} / n$ \\
\hline$a / \AA$ & $9.7523(9)$ & $10.367(4)$ & $12.8489(12)$ \\
\hline$b / \AA$ & $12.5920(11)$ & $13.252(5)$ & $10.5838(10)$ \\
\hline$c / \AA$ & $14.2855(13)$ & $14.439(6)$ & $15.3063(14)$ \\
\hline$\beta /^{\circ}$ & & & $109.4070(10)$ \\
\hline$V / \AA^{3}$ & $1754.3(3)$ & 1983.7(13) & 1963.2(3) \\
\hline$Z$ & 4 & 4 & 4 \\
\hline$\rho_{\text {calcd }} / \mathrm{g} \mathrm{cm}^{-3}$ & 1.591 & 1.468 & 1.483 \\
\hline$F(000)$ & 840 & 904 & 904 \\
\hline reflections collected & 10313 & 11465 & 10614 \\
\hline independent reflections & 4052 & 4428 & 4120 \\
\hline parameters & 212 & 303 & 315 \\
\hline$R$ (int) & 0.0200 & 0.0418 & 0.0196 \\
\hline$R_{1}^{a}, R_{\mathrm{w}}^{b}(I>2 \sigma)$ & $0.0158,0.0365$ & $0.0322,0.0850$ & $0.0471,0.1315$ \\
\hline$R_{1}^{a}, R_{\mathrm{w}}^{b}$ (all data) & $0.0166,0.0368$ & $0.0343,0.0867$ & $0.0506,0.1340$ \\
\hline goodness of fit & 1.052 & 1.038 & 0.954 \\
\hline$\Delta \rho_{\max , \min } / \mathrm{e} \AA^{-3}$ & $0.307,-0.210$ & $0.273,-0.200$ & $0.428,-0.423$ \\
\hline
\end{tabular}


Table S2. Cartesian coordinates (in ångstroms) and energies (in Hartrees) of the CAM-B3LYP/6-311++G(d,p) optimized geometries of $\left[6^{\prime}-\mathbf{M C}\right]^{+}$and $\left[6^{\prime}-\mathrm{SP}\right]^{+}$.

\begin{tabular}{|c|c|c|c|c|c|c|}
\hline \multirow[b]{2}{*}{ Cartesian coordinates } & \multicolumn{3}{|l|}{$\left[6^{\prime}-\mathrm{MC}^{+}\right.$} & \multicolumn{3}{|c|}{$\left.6^{\prime}-\mathrm{SP}\right]^{+}$} \\
\hline & & & & & & \\
\hline $\mathrm{N}$ & -0.06542 & -0.16654 & -2.40533 & -0.537236 & 1.071448 & -1.542922 \\
\hline $\mathrm{C}$ & -1.17072 & 0.304803 & -3.1671 & 0.68088 & 1.584513 & -2.038898 \\
\hline $\mathrm{C}$ & -1.35794 & 0.274861 & -4.5364 & 0.954679 & 2.861281 & -2.489452 \\
\hline $\mathrm{C}$ & -2.54587 & 0.810759 & -5.02032 & 2.247078 & 3.120143 & -2.946368 \\
\hline $\mathrm{C}$ & -3.49455 & 1.349332 & -4.15795 & 3.221031 & 2.133195 & -2.943593 \\
\hline $\mathrm{C}$ & -3.2803 & 1.366076 & -2.78276 & 2.92201 & 0.849613 & -2.484223 \\
\hline $\mathrm{C}$ & -2.10342 & 0.835828 & -2.29156 & 1.647461 & 0.585234 & -2.031347 \\
\hline $\mathrm{C}$ & -1.59047 & 0.709153 & -0.87496 & 1.014666 & -0.703458 & -1.545668 \\
\hline $\mathrm{C}$ & -0.2451 & 0.031147 & -1.11031 & -0.277118 & -0.131989 & -0.867273 \\
\hline $\mathrm{C}$ & 0.745637 & -0.37751 & -0.17983 & -1.44654 & -1.059263 & -0.788121 \\
\hline $\mathrm{C}$ & 0.669061 & -0.22275 & 1.167976 & -2.001823 & -1.498632 & 0.337083 \\
\hline $\mathrm{C}$ & 1.670096 & -0.63819 & 2.107689 & -1.507129 & -1.060246 & 1.631124 \\
\hline $\mathrm{C}$ & 2.925794 & -1.29652 & 1.707748 & -0.421373 & -0.152549 & 1.652929 \\
\hline $\mathrm{C}$ & 3.802231 & -1.63627 & 2.819272 & 0.04259 & 0.313768 & 2.894742 \\
\hline $\mathrm{C}$ & 3.472165 & -1.36295 & 4.091195 & -0.545804 & -0.135387 & 4.040211 \\
\hline $\mathrm{N}$ & 2.282461 & -0.74365 & 4.419066 & -1.573248 & -1.014306 & 4.010921 \\
\hline $\mathrm{C}$ & 1.430202 & -0.40406 & 3.44398 & -2.045087 & -1.459746 & 2.825706 \\
\hline $\mathrm{C}$ & 1.114913 & -0.79346 & -2.99761 & -1.621375 & 1.951125 & -1.156555 \\
\hline $\mathrm{C}$ & -1.41494 & 2.101367 & -0.24012 & 0.627305 & -1.558983 & -2.765542 \\
\hline $\mathrm{C}$ & -2.52996 & -0.18092 & -0.04023 & 1.882842 & -1.527223 & -0.599203 \\
\hline $\mathrm{O}$ & 3.22244 & -1.54437 & 0.544636 & 0.161428 & 0.257634 & 0.570451 \\
\hline $\mathrm{H}$ & -0.62923 & -0.14133 & -5.21843 & 0.205031 & 3.641902 & -2.489228 \\
\hline $\mathrm{H}$ & -2.73196 & 0.806807 & -6.08661 & 2.49132 & 4.112708 & -3.304152 \\
\hline $\mathrm{H}$ & -4.41081 & 1.760064 & -4.56238 & 4.218222 & 2.359145 & -3.29883 \\
\hline $\mathrm{H}$ & -4.02687 & 1.787921 & -2.12047 & 3.685845 & 0.080446 & -2.485695 \\
\hline $\mathrm{H}$ & 1.637185 & -0.85292 & -0.56197 & -1.841437 & -1.36849 & -1.746758 \\
\hline $\mathrm{H}$ & -0.20502 & 0.248528 & 1.604112 & -2.846563 & -2.176544 & 0.30501 \\
\hline $\mathrm{H}$ & 4.110902 & -1.60832 & 4.929267 & -0.218354 & 0.185515 & 5.019389 \\
\hline $\mathrm{H}$ & 1.212826 & -1.81923 & -2.64339 & -1.342351 & 2.606975 & -0.323879 \\
\hline $\mathrm{H}$ & 2.010069 & -0.23231 & -2.73232 & -2.490384 & 1.359398 & -0.870545 \\
\hline $\mathrm{H}$ & 1.007491 & -0.79887 & -4.07683 & -1.90861 & 2.570216 & -2.006155 \\
\hline $\mathrm{H}$ & -0.73394 & 2.721101 & -0.82497 & -0.043399 & -1.022404 & -3.438523 \\
\hline $\mathrm{H}$ & -2.38255 & 2.603535 & -0.20798 & 1.530625 & -1.802189 & -3.325227 \\
\hline $\mathrm{H}$ & -1.03413 & 2.039998 & 0.778845 & 0.158305 & -2.499235 & -2.465942 \\
\hline $\mathrm{H}$ & -2.63886 & -1.17041 & -0.48621 & 2.280742 & -0.927458 & 0.217281 \\
\hline $\mathrm{H}$ & -3.51745 & 0.28037 & -0.00016 & 2.72608 & -1.942915 & -1.152724 \\
\hline $\mathrm{H}$ & -2.17389 & -0.3025 & 0.982179 & 1.32317 & -2.370813 & -0.185664 \\
\hline $\mathrm{H}$ & 4.740083 & -2.12218 & 2.587649 & 0.867059 & 1.011687 & 2.934911 \\
\hline $\mathrm{C}$ & 1.987224 & -0.47559 & 5.828989 & -2.220488 & -1.441283 & 5.26314 \\
\hline $\mathrm{H}$ & 2.749347 & 0.179452 & 6.250781 & -1.505758 & -1.362996 & 6.0784 \\
\hline $\mathrm{H}$ & 1.959041 & -1.41086 & 6.387826 & -2.535471 & -2.478017 & 5.169745 \\
\hline $\mathrm{H}$ & 1.018945 & 0.012493 & 5.907424 & -3.083674 & -0.809116 & 5.470097 \\
\hline $\mathrm{H}$ & 0.515263 & 0.078103 & 3.767553 & -2.875143 & -2.152705 & 2.871347 \\
\hline \multicolumn{7}{|l|}{ Energies } \\
\hline Energy & -920.913607 & & & -920.907146 & & \\
\hline Zero-point energy & 0.364498 & & & 0.365329 & & \\
\hline Enthalpy & -920.530926 & & & -920.524144 & & \\
\hline Gibbs energy & -920.602625 & & & -920.592197 & & \\
\hline
\end{tabular}


Table S3. Cartesian coordinates and energies of the CAM-B3LYP/6-311++G(d,p) optimized geometries of [5'$\mathbf{M C}]^{+}$and $\left[5^{\prime}-\mathbf{S P}\right]^{+}$.

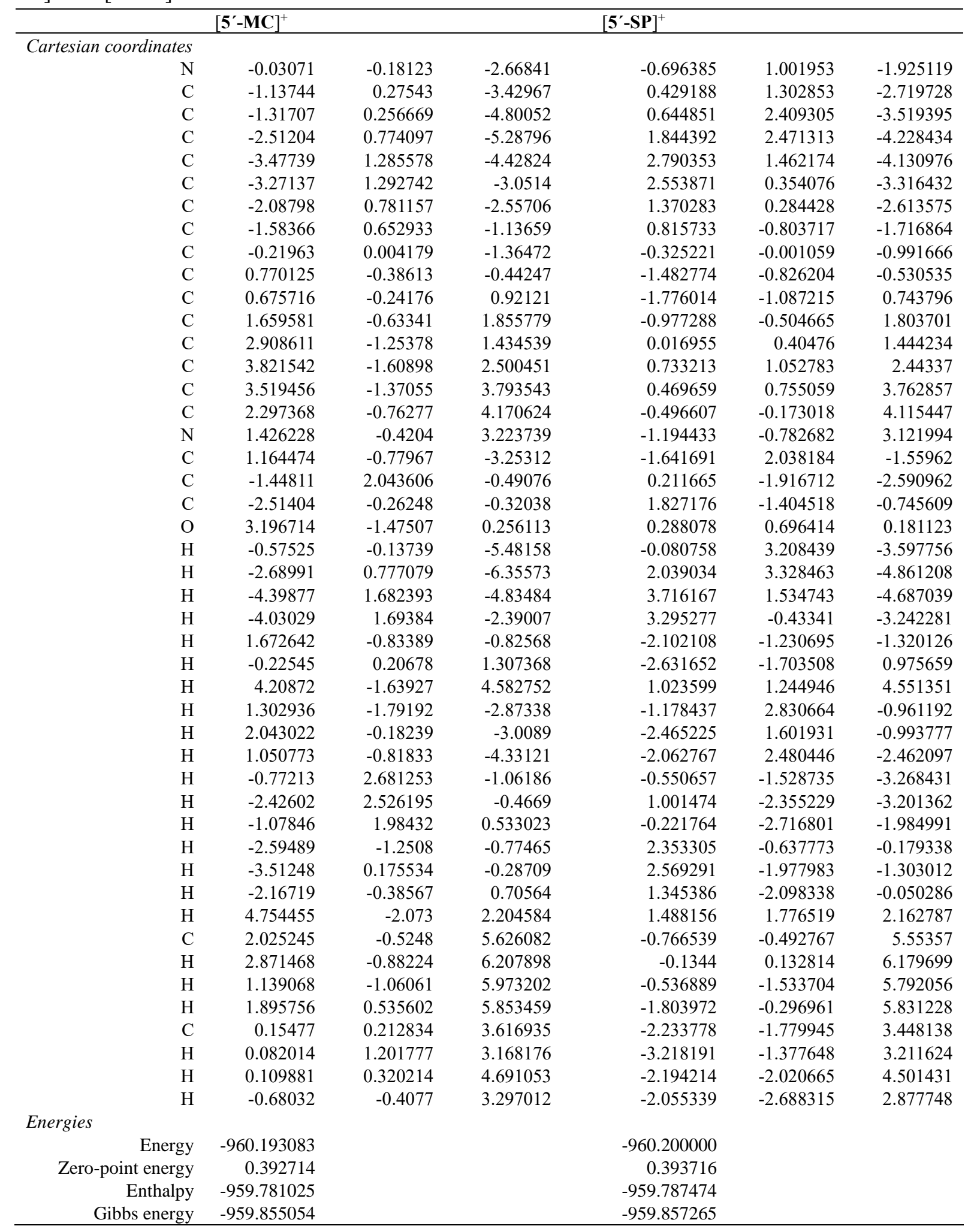


Table S4. Electronic excitation energies, oscillator strengths $(f)$, and compositions of $\left[\mathbf{6}^{\prime}-\mathbf{M C}\right]^{+}$and $\left[\mathbf{5}^{\prime}-\mathbf{M C}\right]^{+}$ calculated by TD-DFT calculations at the CAM-B3LYP/def2-SVP/CPCM $\left(\mathrm{CHCl}_{3}\right)$ level of theory.

\begin{tabular}{lrrrll}
\hline & Energy $(\mathrm{eV})$ & $\lambda(\mathrm{nm})$ & \multicolumn{1}{c}{$f$} & Composition & \\
\hline$\left[\mathbf{6}^{\prime}-\mathbf{M C}\right]^{+}$ & 5.19 & 238.76 & 0.0414 & HOMO-4 => LUMO & $78 \%$ \\
& 4.95 & 250.45 & 0 & HOMO-2 => LUMO+1 & $79 \%$ \\
4.66 & 265.98 & 0.0834 & HOMO-3 => LUMO & $80 \%$ \\
4.43 & 279.66 & 0.0535 & HOMO-1 => LUMO & $88 \%$ \\
& 3.70 & 335.52 & 0 & HOMO-2 => LUMO & $87 \%$ \\
& 3.30 & 375.65 & 1.2228 & HOMO => LUMO & $95 \%$ \\
& & & & & \\
{$\left[\mathbf{5}^{\prime}-\mathbf{M C}^{+}\right.$} & 5.07 & 244.46 & 0.0706 & HOMO-4 => LUMO & $72 \%$ \\
& 4.71 & 263.20 & 0.0473 & HOMO => LUMO+1 & $86 \%$ \\
& 4.55 & 272.42 & 0.0555 & HOMO-3 => LUMO & $83 \%$ \\
& 4.26 & 291.04 & 0.0772 & HOMO-1 => LUMO & $88 \%$ \\
& 3.13 & 396.67 & 0.0005 & HOMO-2 => LUMO & $94 \%$ \\
& 2.74 & 451.86 & 1.3289 & HOMO => LUMO & $95 \%$ \\
\hline
\end{tabular}

\section{References}

S1 D. Bialas, A. Zitzler-Kunkel, E. Kirchner, D. Schmidt, F. Würthner, Nat. Commun. 2016, 7, 12949. 